\title{
The heat shock protein 90 of Toxoplasma gondii is essential for invasion of host cells and tachyzoite growth
}

\author{
Hongchao Sun ${ }^{1}$, Xunhui Zhuo ${ }^{1}$, Xianfeng Zhao ${ }^{2}$, Yi Yang ${ }^{1}$, Xueqiu Chen ${ }^{1}$, Chaoqun Yao ${ }^{3}$, \\ and Aifang $\mathrm{Du}^{1, *}$ \\ ${ }^{1}$ Institute of Preventive Veterinary Medicine \& Zhejiang Provincial Key Laboratory of Preventive Veterinary Medicine, College of \\ Animal Sciences, Zhejiang Provincial Key Laboratory of Preventive Veterinary Medicine, Zhejiang University, Hangzhou 310058 , \\ PR China \\ 2 Shenzhen Entry-exit Inspection and Quarantine Bureau, Shenzhen 518045, PR China \\ 3 Department of Biomedical Sciences and One Health Center for Zoonoses and Tropical Veterinary Medicine, Ross University School of \\ Veterinary Medicine, P.O. Box 334, Basseterre, St. Kitts, West Indies
}

Received 24 February 2017, Accepted 25 May 2017, Published online 19 June 2017

\begin{abstract}
Toxoplasma gondii is an obligate intracellular apicomplexan parasite that infects almost all warmblooded vertebrates. Heat shock proteins (HSP) regulate key signal transduction events in many organisms, and heat shock protein 90 (Hsp90) plays an important role in growth, development, and virulence in several parasitic protozoa. Here, we discovered increased transcription of the $H s p 90$ gene under conditions for bradyzoite differentiation, i.e. alkaline and heat shock conditions in vitro, suggesting that $H s p 90$ may be connected with bradyzoite development in $T$. gondii. A knockout of the $T g H s p 90$ strain $(\Delta H s p 90)$ and a complementation strain were constructed. The $T g H s p 90$ knockout cells were found to be defective in host-cell invasion, were not able to proliferate in vitro in Vero cells, and did not show long-time survival in mice in vivo. These inabilities of the knockout parasites were restored upon complementation of $T g H s p 90$. These data unequivocally show that $T g H s p 90$ contributes to bradyzoite development, and to invasion and replication of T. gondii in host cells.
\end{abstract}

Key words: Toxoplasma gondii, Heat shock protein 90, Invasion, Differentiation, Replication.

\begin{abstract}
Résumé - La protéine de choc thermique 90 de Toxoplasma gondii est essentielle pour l'invasion des cellules hôtes et la croissance des tachyzoïtes. Toxoplasma gondii est un Apicomplexa parasite intracellulaire obligatoire qui infecte presque tous les vertébrés à sang chaud. Les protéines de choc thermique régulent les événements principaux de transduction du signal chez de nombreux organismes et la protéine de choc thermique 90 (Hsp90) joue un rôle important dans la croissance, le développement et la virulence chez plusieurs protozoaires parasites. Dans ce travail nous avons découvert une augmentation de la transcription du gène de $H s p 90$ dans les conditions de différenciation des bradyzoïtes et de chocs alcalins et thermiques in vitro, ce qui suggère que Hsp90 est lié au développement des bradyzoïtes chez $T$. gondii. Un knock-out de la souche $\operatorname{TgHsp} 90$ ( $\Delta H s p 90)$ et une souche de complémentation ont été construits. Les cellules knock-out $T g H s p 90$ se sont révélées défectueuses dans l'invasion des cellules hôtes, n'ont pas pu proliférer in vitro dans des cellules Vero et n'ont pas montré de survie de longue durée chez la souris in vivo. Ces défauts des parasites knock-out ont été restaurés lors de la complémentation de $T g H s p 90$. Ces données montrent sans équivoque que $T g H s p 90$ contribue au développement des bradyzoïtes, et à l'invasion et à la réplication de $T$. gondii dans les cellules hôtes.
\end{abstract}

\section{Introduction}

Toxoplasma gondii is a protozoan parasite of medical and veterinary importance. It causes toxoplasmosis in almost all homoeothermic animals, including humans, leading to potentially severe congenital infections and life-threatening

\footnotetext{
*Corresponding author: afdu@zju.edu.cn
}

conditions in the developing fetus or newborn [17, 31]. There are three infective stages of $T$. gondii including the rapidly replicating tachyzoite, the slow-replicating bradyzoite, and the sporozoite-containing oocyst. The first two are asexual replication stages in the intermediate host of most, if not all, warm-blood animals including humans. The latter is the result of the sexual replication that occurs in the definitive hosts, i.e. cats and other felids $[23,41]$. 
Humans become infected by eating tissue cysts in undercooked meat, consuming oocyst-contaminated food or water, or accidentally ingesting oocysts in cat feces [26]. The ability of T. gondii to cycle between the tachyzoite and bradyzoite stages in intermediate hosts is the key to its survival and a major factor in the pathogenesis of toxoplasmosis [12]. Although the cycle between tachyzoite and bradyzoite is critical to parasite survival, the underlying molecular mechanisms remain largely unknown to date [39, 46, 54].

Heat shock protein (HSP) families are evolutionarily conserved in many organisms throughout various taxa [30] and were first discovered in Drosophila in 1962 [25]. They are important molecular chaperones for maintaining cellular functions to prevent proteins from misfolding and aggregation in crowded surroundings [19, 50]. Their expression levels increase dramatically when the cells are cultured under stress conditions [28] such as heat shock, alkaline treatment, and some chemical reagents. In the past several years, a number of research groups have shown that the HSPs are not only involved in protein modification and folding but also participate in many signaling pathways [40]. Furthermore, HSPs affect the immune system, such as binding antigen during antigen processing, and induce cytokine release [6].

Among all HSPs, HSP90, an ATP-dependent protein, has received widespread attention in cancer research because of its important role in carcinogenesis [25]. HSP90 also regulates human immunodeficiency virus (HIV-1) reactivation by mitogen-activated protein kinase/extracellular signal-regulated kinase and Protein Kinase C (PKC) pathways (MAPK/ERK/ $\mathrm{PKC})$ to influence replication and gene expression of HIV-1 virus [1, 42]. Although HSP90 has been targeted for anticancer therapy in the past decades, little research has been carried out on this protein in the protozoan parasites, a diverse group of unicellular organisms that affect more than 500 million people in the world $[15,35,44]$. It has been described in the last few years in a handful of apicomplexan parasites such as Plasmodium falciparum [3], Babesia gibsoni [53], Theileria annulata [34], Eimeria tenella [38], and T. gondii [2, 13]. The role of Hsp90 in growth and stage conversions between tachyzoites and bradyzoites had been described using geldanamycin (GA), but gene deletion and complementation were not involved in the research [13]. The main purpose of the present study was to illustrate the cellular functions of HSP90 in T. gondii. A knockout of the Hsp90 gene was generated. We showed that $T$. gondii HSP90 was involved in bradyzoite development, played an important role in invasion and growth of the parasite in vitro, and was associated with virulence in $\mathrm{BALB} / \mathrm{c}$ mice in vivo.

\section{Materials and methods}

\section{Ethics}

All mice were treated in strict accordance with the recommendations of the Guide for the conduct of studies with experimental animals of the People's Republic of China. The use of animals was approved by Zhejiang University Experimental Animal Ethics Committee (Permit Number:
ZJU201308-1-10-072). All efforts were made to minimize numbers and to reduce suffering of the animals used.

\section{Host cell and parasite cultures}

The T. gondii RH $\Delta k u 80$ strain was maintained on monolayers of African green monkey kidney (Vero) cells at $37^{\circ} \mathrm{C}$ and $5 \% \mathrm{CO}_{2}$. The culture medium was Dulbecco's Modified Eagle's Medium (DMEM, HyClone) supplemented with 10\% fetal calf serum (FCS, HyClone), $2 \mathrm{mM}$ L-glutamine, and 100 units penicillin/100 mg streptomycin [55].

\section{Generation of Hsp90 gene knockout parasite}

The parental strain used to generate the knockout strain was RH $\Delta k u 80$. Deletion of the Ku80 gene involved in DNA repair via the non-homologous and joining pathway results in greater levels of homologous recombination, allowing for incorporation of reporter proteins into the $3^{\prime}$ end of the endogenous genes [43]. The knockout vector construct was engineered with the selectable marker ble, resistant to phleomycin using the pBluescript SK (+) [33]. RH $\Delta k u 80$ genomic DNA was isolated using the TIANGEN genomic DNA isolation kit by following the manufacturer's protocol (TIANGEN, Beijing). Briefly, $\sim 1800 \mathrm{bp}$ of the $5^{\prime}$ flanking and $3^{\prime}$ flanking sequences of the TgHsp90 (GenBank ID 288380) gene were amplified using the RH $\Delta k u 80$ genomic DNA as templates by polymerase chain reaction (PCR). The primers were $\mathrm{P} 1 / \mathrm{P} 2$ and $\mathrm{P} 3 / \mathrm{P} 4$, listed in Table 1 . Then, the products were flanked with KpnI and HindIII, SpeI and NotI (TAKARA, Dalian) and cloned into the pBluescript SK $(+)$ (Promega, USA) vector. After linearization with NotI, $25-50 \mu \mathrm{g}$ of the plasmid was electroporated into the RH $\Delta k u 80$ strain by a Bio-Rad electroporation system; the electroporation conditions were $1.5 \mathrm{kV}, 0.5 \mathrm{~ms}$ for three times. Transfected parasites were selected with phleomycin (Sigma, USA), high-dose extracellular treatment $(50 \mu \mathrm{g} / \mathrm{mL})$, and low-dose treatment $(5 \mu \mathrm{g} / \mathrm{mL})$ during growth in Vero cells. After a second round of selection, the individual clones were obtained by limiting dilution. The positive clones were confirmed by PCR using primers P5/P6 and P7/P8, listed in Table 1, as well as determined by Western blotting analysis.

\section{Generation of Hsp90 complemented parasites}

To further confirm the role of $T g H s p 90$, the $T g H s p 90$ gene was reintroduced into the $\Delta H s p 90$ knockout parasites. The HSP90 sequence (GenBank ID AY344115.1) was amplified by PCR using primers P9/P10. Afterwards, surface antigen 1 (SAG1) promoter and granule 1 (GRA1) poly A signal sequences were added to the $5^{\prime}$ and $3^{\prime}$ end, respectively. The construct was then cloned into the pTCY vector. The vector was kindly offered by Professor Liuqun of the China Agricultural University College of Veterinary Medicine. The vector contained the chloramphenicol resistance gene CAT. We transformed the product to Escherichia coli (top10) cells and the plasmids were extracted by plasmid extraction kit (Axygen, America); 20-50 $\mu \mathrm{g}$ of the plasmid was linearized with 
Table 1. Primers used in this study.

\begin{tabular}{ll}
\hline Primers & Sequences \\
\hline P1 & 5'-GGTACCAGAGGCCTTCAGCTTCGCGGAGA-3' \\
P2 & 5'-AAGCTTCTTGTCTCGAGCGAGGAGAGTT-3' \\
P3 & 5'-ACTAGTGCAGCTTCCAATGTCACCCG-3' \\
P4 & 5'-GCGGCCGCTGTCAAATACGAAGTTCA \\
& GCCTCTC-3' \\
P5 & 5'-CAGAGAGCAGCGCAGAGAGAACGG-3' \\
P6 & 5'-TTAAGAGATGCCTGCAAGCAATTCG-3' \\
P7 & 5'-ATGCATGACCAAGCGACGCCCAAC-3' \\
P8 & 5'-GGTGACGCTTCTCGCTTTCGCT-3' \\
P9 & 5'-CATATGGCGGACACCGAGACCTTC-3' \\
P10 & 5'-AAGCTTGTCGACCTCCTCCATCTTCGAGGT-3' \\
Actin-F & 5'-CACGAGAGAGGATACGGCTTCACCA-3' \\
Actin-R & 5'-CCATCGGGCAATTCATAGGACTTCTC-3' \\
B1-F & 5'-GGAACTGCATCCGTTCATGAG-3' \\
B1-R & 5'-TCTTTAAAGCGTTCGTGGTC-3' \\
\hline
\end{tabular}

$K p n I$ and transfected into the $\Delta H s p 90$ parasite. Stable clones were selected by $20 \mu \mathrm{g} / \mathrm{mL}$ chloramphenicol (Sigma, USA). HSP90 expression was confirmed by Western blotting.

\section{Preparation of anti-rTgHsp90 polyclonal antibody and Western blotting}

The coding region of $T g \mathrm{Hsp} 90$ was cloned into the pMD$18 \mathrm{~T}$ vector followed by cloning into the $\mathrm{pET}-28 \mathrm{a}$ vector and transfection into the Escherichia coli BL21 (DE3) strain (TAKARA, Dalian) for expression. The recombination protein was purified by affinity chromatography using Ni-IDA agarose (QIAGEN, Germany) and then assessed by sodium dodecyl sulfate-polyacrylamide gel electrophoresis (SDS-PAGE). The concentration of the protein was measured by the bicinchoninic acid (BCA) protein assay kit (Beyotime, Shanghai). The protein was subcutaneously injected into the test rabbits. For the first immunization, the protein concentration was $0.5 \mathrm{mg} / \mathrm{kg}$ emulsified with Freund's complete adjuvant. The second and third immunizations were performed every two weeks; the protein concentration was $0.25 \mathrm{mg} / \mathrm{kg}$ emulsified with Freund's incomplete adjuvant. Anti- $T g H s p 90$ antibodies were obtained from immunized rabbits, and the titers were determined by enzyme-linked immunosorbent assay (ELISA) using recombination antigen at 13 days after the last immunization.

The parasites $\left(10^{6}\right)$ were collected and centrifuged at $3000 \times \mathrm{g}$ for $5 \mathrm{~min}$; the pellet was resuspended in cold phosphate-buffered saline (PBS) and passed three times through a 30-gauge needle syringe [14]. Purified parasites $\left(10^{6}-10^{7}\right)$ were lysed with radioimmunoprecipitation assay (RIPA) buffer (Beyotime) in the presence of $1 \mathrm{mM}$ phenylmethanesulfonyl fluoride (PMSF) as a protease inhibitor (Beyotime). Total proteins were quantified by the BCA protein assay kit (Beyotime); 15-20 $\mu \mathrm{g}$ of total proteins was separated on $12 \%$ SDS-PAGE gels and transferred to nitrocellulose membranes (Axygen, USA) for analysis. Rabbit anti- $\mathrm{TgHsp} 90$ antibody, diluted 1:1000 in 5\% skim milk, which was dissolved in Tris-buffered saline with Tween 20 (TBST) (TBS buffer supplied with $0.05 \%$ Tween 20), was used to detect Hsp90. The nitrocellulose (NC) filter membrane was incubated for
$2 \mathrm{~h}$ at $37{ }^{\circ} \mathrm{C}$ in an incubator. Horseradish peroxidase (HRP)conjugated goat anti-rabbit immunoglobulin $\mathrm{G}$ (IgG), diluted $1: 5000$ in $5 \%$ slim milk/TBST, was used as the secondary antibody. Finally, the signals were detected by electrochemiluminescence (ECL) (CYANAGEN, Italy).

\section{Real-time PCR}

Many stressful conditions have been connected with the development of the $T$. gondii bradyzoite. One such condition

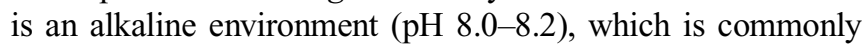
applied in studies on the in vitro differentiation of $T$. gondii. Heat shock is also often used [16]. We detected the expression level of Hsp90 in order to analyze whether T. gondii HSP90 played a role when bradyzoites were induced under stress by alkaline $\mathrm{pH}$ or heat shock. Then, T. gondii bradyzoite genes BAG1 and MAG1 were analyzed by real-time PCR among $\mathrm{RH} \Delta k u 80, \Delta H s p 90$, and Hsp90-complementation strains to further test whether $H s p 90$ plays a role during bradyzoite differentiation. The relative expression level of $H s p 90$ transcripts was determined by real-time RT-PCR using actin transcripts as the internal control. Primers for $T$. gondii actin are shown in Table 1 ; the values were calculated by the $2^{-\Delta \Delta C T}$ method.

\section{Immunofluorescence and invasion assays}

To test the invasion of host cells, two-color (red/green) invasion assays were performed, as previously described [5]. Red/green invasion assays were performed as described for indirect immunofluorescence. RH $\Delta k u 80, \Delta H s p 90$, and complemented parasites were seeded on monolayers of Vero cells on coverslips in 12 -well plates with $1 \times 10^{5} /$ well. After 1,2 , and $4 \mathrm{~h}$, the coverslips were washed with sterile PBS, and then fixed with $4 \%$ formaldehyde for $15 \mathrm{~min}$, and external (attached) parasites were stained with rabbit antibody (SAG3), followed by washing. Monolayers were permeabilized with $0.3 \%$ Triton X-100, and internal (invaded) parasites were incubated with mouse mAb 4D-5 (SAG3), a membrane surface antigen of the SAG family in $T$. gondii generated in the laboratory. Its specificity was confirmed by specific reaction to positive porcine serum for $T$. gondii, but not to porcine serum of the animals infected with Cryptosporidium suis, Mycoplasma suis, Streptococcus suis, Salmonella choleraesuis, Cysticercus cellulosae, Isospora suis, or Trichinella spiralis kept in our laboratory (data not shown). Secondary antibodies were Alexa Fluor 488 (Green) and Alexa Fluor 568 (Red) (Life Technologies, USA), 4',6-diamidino-2-phenylindole (DAPI) was added to the secondary antibody solution to stain host nuclei. Finally, the images were obtained using an Olympus confocal microscope (IX71) with $60 \times$ magnification and the numbers of intracellular parasites (green), extracellular parasites (red), and host-cell nuclei (blue) were counted. Mean values of three independent experiments were determined.

\section{Intracellular growth}

Equal numbers $\left(10^{5}\right)$ of RH $\Delta k u 80, \Delta H s p 90$, and complemented parasites were used to infect monolayers of Vero cells 


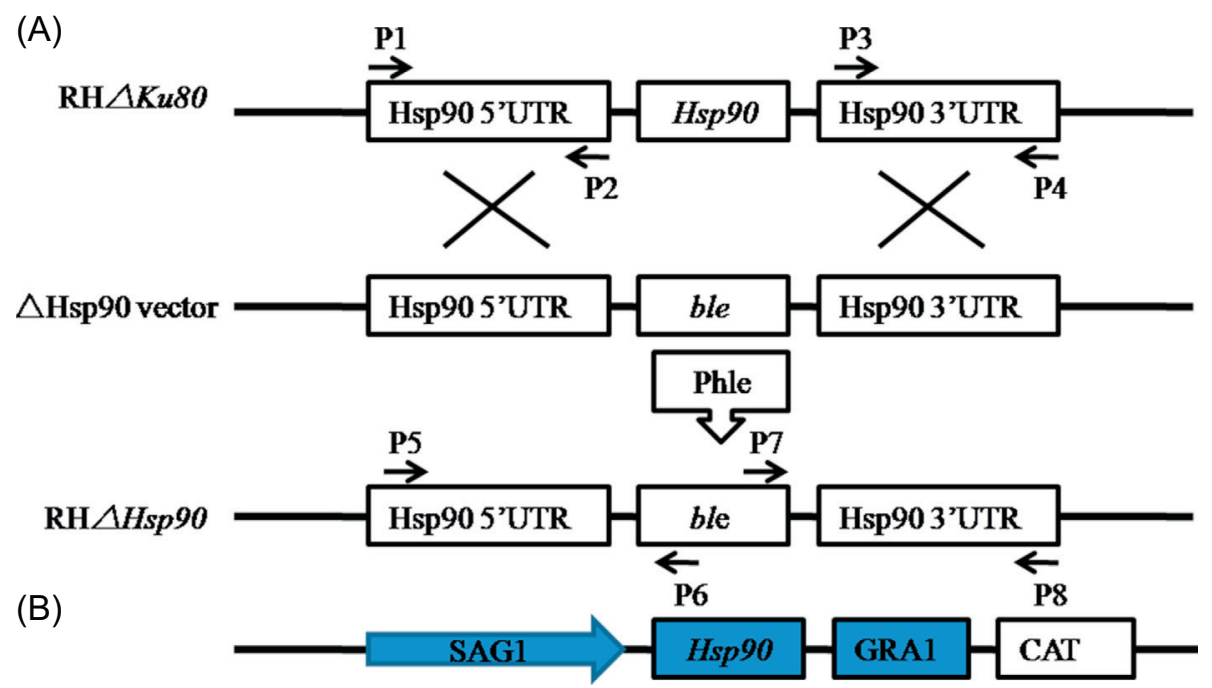

Figure 1. Generation of $T g H s p 90$ knockout $(\Delta H s p 90)$ and complementation parasites. (A) Schematic illustration of the strategy used to generate the $\mathrm{TgHsp} 90$ knockouts by homologous recombination. The Hsp90 coding region was replaced by the selectable marker Ble; the knockout vector was transfected into the RH $\Delta k u 80$ strain and selected by phleomycin. (B) Diagram of genetic complementation of Hsp90. An $H s p 90$ coding sequence and the $C A T$ selection marker, which was flanked by $T$. gondii Tublin promoter and $3^{\prime}$ poly A signal (provided by Professor Liuqun, College of Veterinary Medicine, China Agricultural University), was transfected into the $T g H s p 90$ defective strain to generate the complemented strain.

grown on six-well plates; the infection ratio was 1:1. The noninvasive tachyzoites were removed after infection for $2 \mathrm{~h}$ and fresh medium was added to the cells. Growth of the parasites was then observed by inverted microscope. Next, 24, 48, 72, and $96 \mathrm{~h}$ post-infection (PI), the parasites were collected and genomic DNA was extracted using the TIANGEN genomic DNA isolation kit by following the manufacturer's protocol (TIANGEN, Beijing). The standard curve was obtained by the known concentration of the $\mathrm{RH} \Delta k u 80$ parasites via SYBR-green real-time PCR using B1 gene primers, and the parasite numbers were calculated by interpolation from this standard curve [8]. Triplicates were used for each time point of three independent experiments.

\section{In vivo virulence of $\Delta H s p 90$ knockout in mouse}

Female BALB/c mice 6-8 weeks of age were infected intraperitoneally (IP) with $10^{3}$ cells of parental RH $\Delta k u 80$, $\triangle H$ sp 90 knockout, or complemented parasites. In each experiment, 12 mice were used for each type of parasite. The mice were monitored daily for survival until 28 days PI when experiments ended. This experiment was repeated three times. Survival rates were calculated from all three independent experiments.

The tissues of the liver, spleen, lungs, and brain were collected from the mice that showed clinical symptoms but had not died. We collected the organs using sterile scissors and divided them into masses of equal quality of $0.5 \mathrm{mg}$ each. Genomic DNA was extracted from each tissue using a genomic DNA extraction kit (TIANGEN, Beijing). T. gondii DNA was detected by SYBR-green real-time PCR using B1 primer pairs (Table 1). The standard curve was obtained by the known concentration of the RH $\Delta k u 80$ parasites with the primers (B1), and the parasite number was calculated by interpolation from this standard curve [7]. The results were based on three independent experiments.

\subsection{Statistical analysis}

A Student's $t$-test was performed by SPASS (Statistical Analysis System, Version 16.0). It was considered statistically significant if $p \leq 0.05$.

\section{Results}

\section{Generating TgHsp90 knockout of Toxoplasma gondii}

To understand the biological function of $\operatorname{TgHsp} 90$, the Hsp90 deletion vector was transfected into RH $\Delta k u 80$ parasites of $T$. gondii, which was used as a wild-type strain as far as $T g H s p 90$ was concerned. The $T g H s p 90$ gene was replaced by the coding sequence of the Ble selectable marker gene under the control of the SAG1 promoter. Figure 1A shows a schematic diagram of the targeting construct $H s p 90$, designated as $4 H s p 90$. To identify the knockout clones, PCR analysis was performed using primers (P5/P6 in Table 1) from the $5^{\prime}$ flanking genomic regions combined with the ble gene and the ble gene combined with $3^{\prime}$ flanking genomic regions (P7/P8 in Table 1). The amplification of the fragment confirmed that proper integration was obtained. As shown in Figure 2A, a 529-bp fragment DNA product was successfully amplified from both $\Delta H s p 90$ and RH $\Delta k u 80$ parasites of $T$. gondii. Figure $2 \mathrm{~B}$ shows the PCR results which confirmed that successful deficient parasite was achieved. Western blotting analysis was performed using anti-Hsp90 rabbit antiserum (1:1000), 
(A)

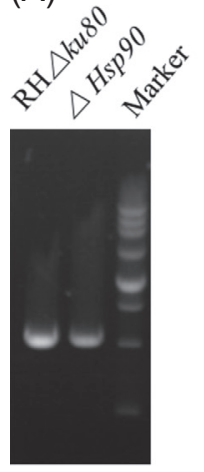

(B)

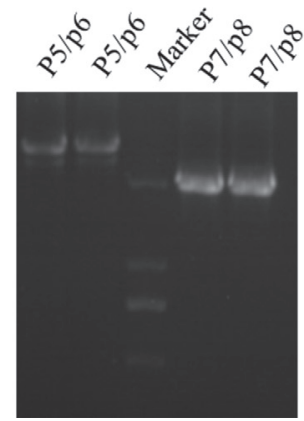

(C)

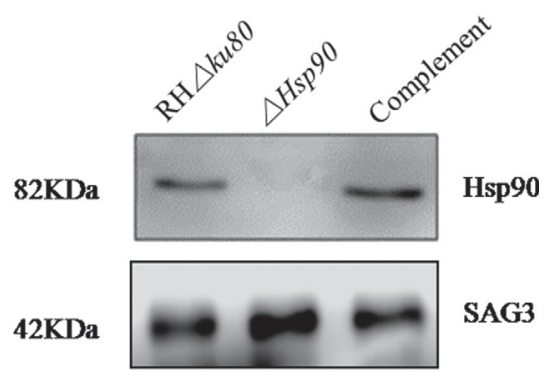

Figure 2. Confirmation of $\Delta H s p 90$ and complemented parasites. (A, B) PCR analysis of $\Delta H s p 90$ strains. The positions of the primers are shown in Figure 1A. P5/P6 and P7/P8 were used to amplify the conjunct regions of $5^{\prime}$ and $3^{\prime}$ integration of the Ble gene construct into the corresponding Hsp90 locus, respectively. (C) Western blotting analysis showing detection of Hsp90 in wild-type T. gondii RH $\Delta k u 80$ and in the complemented strain, but absence in $4 H s p 90$ parasites. Surface antigen (SAG3) was used as a loading control.
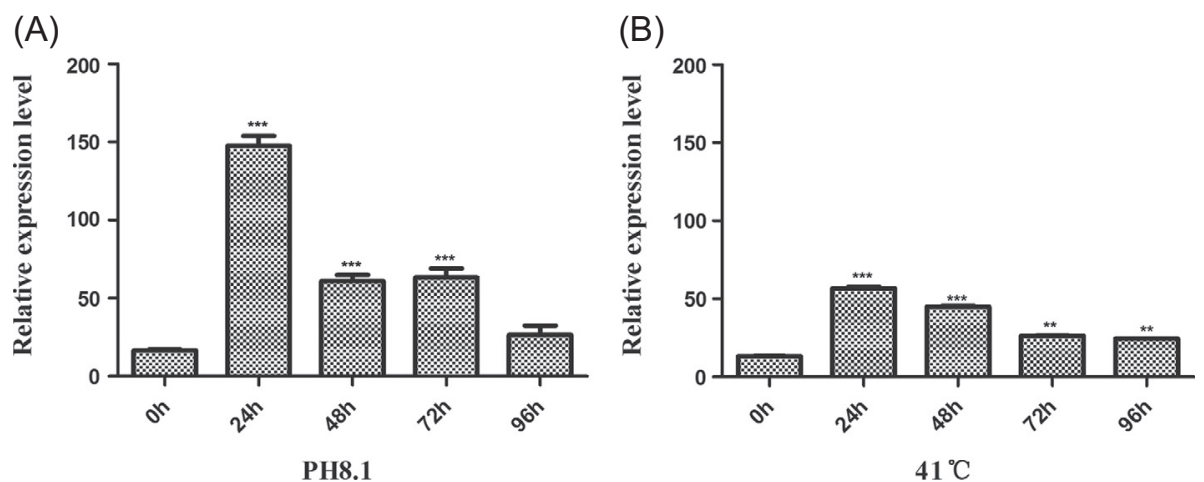

Figure 3. Real-time RT-PCR of Hsp90 transcriptional level during bradyzoite differentiation in vitro. The RH $\Delta k u 80$ T. gondii cells were exposed to alkaline treatment ( $\mathrm{pH} 8.1$ ) (A) or heat shock $\left(41^{\circ} \mathrm{C}\right)(\mathrm{B})$ for $0,24,48,72$ or $96 \mathrm{~h}$. Total RNA was purified from the bradyzoites collected at the end of each time point. The expression levels were determined by real-time RT-PCR using actin as an internal control. Samples collected at $24,48,72$ or $96 \mathrm{~h}$ were compared to those at time $0 \mathrm{~h}$. “***" indicate a statistically significant difference $(p<0.001)$. Values represent mean $\pm S D, n=3$ experiments.

the $4 H s p 90$ knockout parasite had no detectable level of Hsp90 proteins. In contrast, the parental RH $\Delta k u 80$ cells had a high level of expression of the protein (Fig. 2C).

We then generated complemental parasites of $4 H s p 90$ knockout for $H_{s p} 90$ gene complementation (Figure 1B). Individual clones were selected by $20 \mu \mathrm{M}$ chloramphenicol. The expression of HSP90 was detected by Western blotting with anti-Hsp 90 rabbit antiserum (1:1000), showing that the complemental parasites were successfully generated (Fig. 2C).

\section{High expression of T. gondii Hsp90 during bradyzoite differentiation in vitro}

To determine the relative expression level of Hsp90 in in vitro differentiation of $T$. gondii, the RH $\triangle k u 80$ parasites were exposed to alkaline $\mathrm{pH}\left(\mathrm{pH} 8.1,0.03 \% \mathrm{CO}_{2}\right)$ or heat shock $\left(41{ }^{\circ} \mathrm{C}\right)$. The results showed that both alkaline $\mathrm{pH}$ and heat shock induced increasing expression of HSP90. Parasites exposed to alkaline $\mathrm{pH}$ for 24,48 , and $72 \mathrm{~h}$ increased the Hsp90 transcript level by 8.9-, 3.7-, and 3.8-fold in comparison with cells that were not exposed to such conditions at time $0 \mathrm{~h}$ $(p<0.001)$. Similar results were obtained for cells being exposed to heat shock (Fig. 3). Then, the relative expression level of bradyzoite genes BAG1 and MAG1 was detected by SYBR-green real-time PCR from Vero cells infected with $\mathrm{RH} \Delta k u 80, \Delta H s p 90$, and complementary strains. The transcript level of BAG1 $(p<0.05)$ and MAG1 $(p<0.01)$ was significantly decreased when the $T g \mathrm{Hsp} 90$ gene was lacking, compared to the RH $\triangle k u 80$ parasite (Fig. 4). These data unequivocally showed that $T$. gondii HSP90 plays a role when bradyzoites are under stress induced by alkaline $\mathrm{pH}$ or heat shock.

\section{Hsp90 knockout parasites are defective in invasion of host cells}

We subsequently tested whether HSP90 plays a role in host-cell invasion. We used red/green antibody assays to distinguish extracellular parasites from intracellular ones. For the invasion assay, the parasites were incubated with Vero cells 

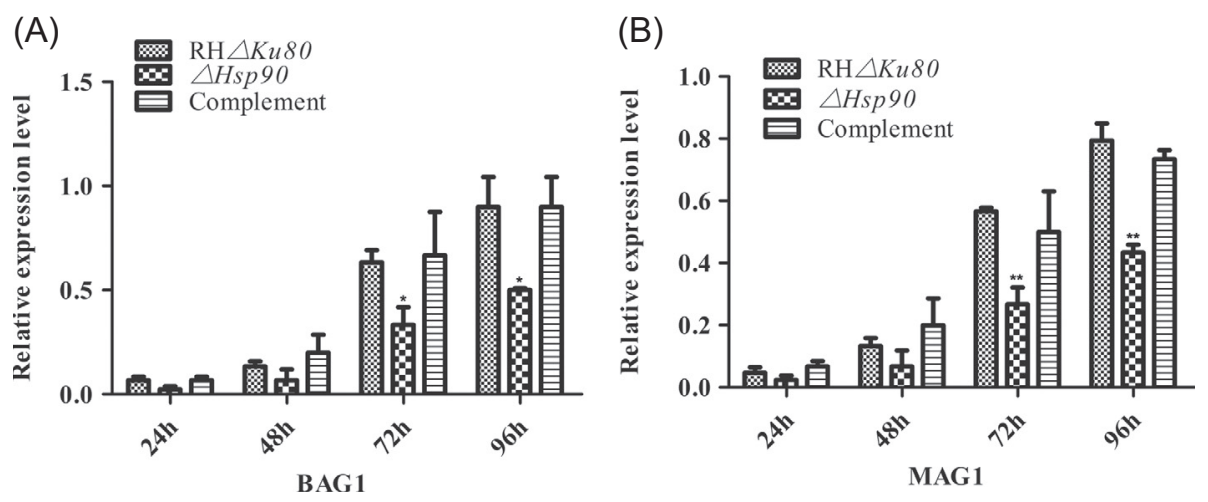

Figure 4. The relative expression level of BAG1 and MAG1. $4 H s p 90, \mathrm{RH} \Delta k u 80$ and complemented parasites $\left(10^{5}\right)$ were cultured in Vero cells for 24, 48, 72 and $96 \mathrm{~h}$. The RNA was extracted by the Trizol dissociation method and detected by relative SYBR-green real-time PCR using actin transcripts as an internal control. ${ }^{*} p<0.05,{ }^{*} p<0.01$. Data represent mean $\pm S D, n=3$ experiments.

for 1,2 , or $4 \mathrm{~h}$. Afterwards, invasion was detected by the twocolor (red/green) antibody test as mentioned above. Compared to that of the RH $\Delta k u 80$ parental parasite, the invasion rate of the $\triangle H S P 90$ knockout in Vero cells was reduced by $57.5 \%$ $(p<0.01), 48.2 \%(p<0.05)$, and $17.5 \%(p<0.05)$ for the three periods of time $(1,2$, and $4 \mathrm{~h})$, respectively. In contrast, the rate of the knockout being complemented with $\operatorname{TgHSP} 90$ was at a level that was similar to that of parental cells (Fig. 5). The invasion ratio assay showed that deletion of Hsp90 caused an early invasion defect of $T$. gondii, and this defect was restored by complementation of the Hsp90 gene locus. Taken together, these data showed that $T g H S P 90$ plays an important role in host-cell invasion.

\section{Hsp90 deletion results in a defective growth phenotype}

To evaluate the growth rate of Hsp90 knockout, $\Delta H s p 90$, $\mathrm{RH} \Delta k u 80$, and complemented parasites were added to monolayer Vero cells at a 1:1 ratio. The parasite plaques were examined using an inverted microscope, and the results showed that the plaque produced by $\triangle P K A R$ strains (Fig. 6B) was significantly smaller than that of $\mathrm{RH} \triangle k u 80$ and complemented parasites (Figs. 6A, 6C). At 24, 48, 72, and $96 \mathrm{~h}$ of incubation, cell cultures were collected and parasite numbers were determined with SYBR-green real-time PCR that amplified specific oligonucleotides of the B1 gene of $T$. gondii. As shown in Figure $6 \mathrm{D}$, the growth of $\triangle H s p 90$ knockout decreased by $58.1 \%, 40.6 \%, 38.3 \%$, and $43.1 \%$ at the time points 24,48 , 72 , and $96 \mathrm{~h}$, respectively, in comparison with that of the RH $\Delta k u 80$ parental cells. The difference was statistically significant at $24 \mathrm{~h}(p<0.001)$. In contrast, the growth of the $\Delta H$ sp 90 knockout complemented with the HSP90 gene was comparable to that of the RH $\Delta k u 80$ parental cells $(p>0.05)$. These data collectively demonstrated that Hsp90 was required for the normal growth of $T$. gondii.

\section{TgHsp90 is essential for virulence in the $\mathrm{RH}$ strain}

The above observation of reduced growth in vitro was intriguing. We next investigated whether Hsp90 contributed

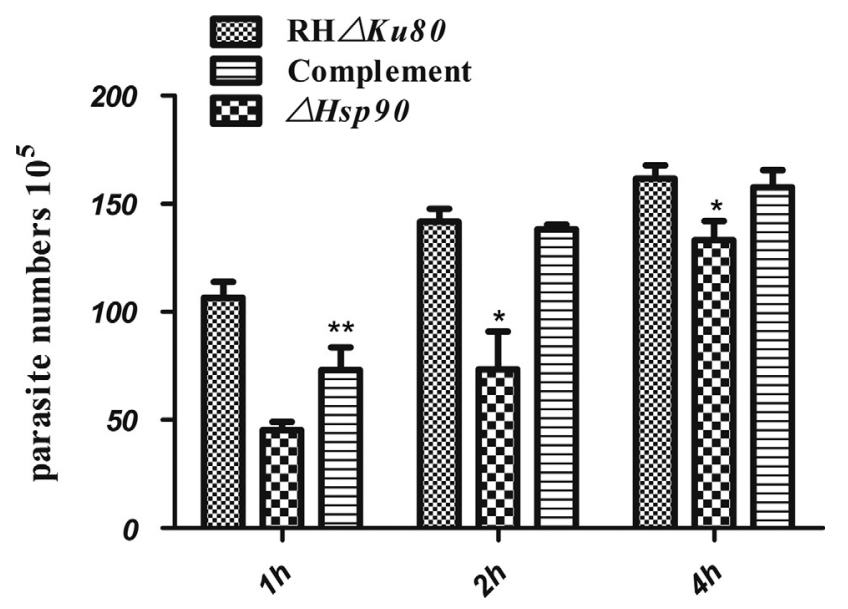

Figure 5. Invasion of the $\Delta H s p 90$ parasite. The invasion ratio of $\triangle H s p 90, \mathrm{RH} \triangle k u 80$ and complemented parasites was evaluated, the points of time were 1, 2 and $4 \mathrm{~h}$, and anti-SAG3 monoclonal antibody was used as the red-green attachment assay. Fields were randomly selected and data were mean $\pm S D$ of three independent experiments. "**" and "** indicate statistically significant difference at $p<0.01$ and $p<0.05$, respectively.

to virulence of the $\mathrm{RH}$ strain in vivo. Equal numbers of $\Delta H s p 90, \mathrm{RH} \Delta k u 80$, and complemented parasites were injected IP to BALB/c mice at a dose of $10^{3}$ parasites/mouse. The mice that received the wild-type parasites developed clinical signs at day 4 PI, and all died between 6 and 9 days PI. In contrast, the mice given the $\triangle H s p 90$ knockout cells showed no clinical signs before 14 days PI, and about a quarter of the mice remained alive even until 28 days PI when the experiment ended (Fig. 7). These results clearly showed that deletion of the $T g \mathrm{HSP} 90$ gene attenuated $T$. gondii and greatly reduced parasite virulence in mice.

We also determined the parasite load in tissues of $T$. gondii infected mice in the liver, spleen, lungs, and brain. The tissues were collected from the animals at the same time point when they were euthanized. SYBR-green real-time PCR was used to quantify parasite loads in each tissue. Results revealed that all tissues were $T$. gondii infection positive, but the most numerous parasites were observed in the brain and liver, 

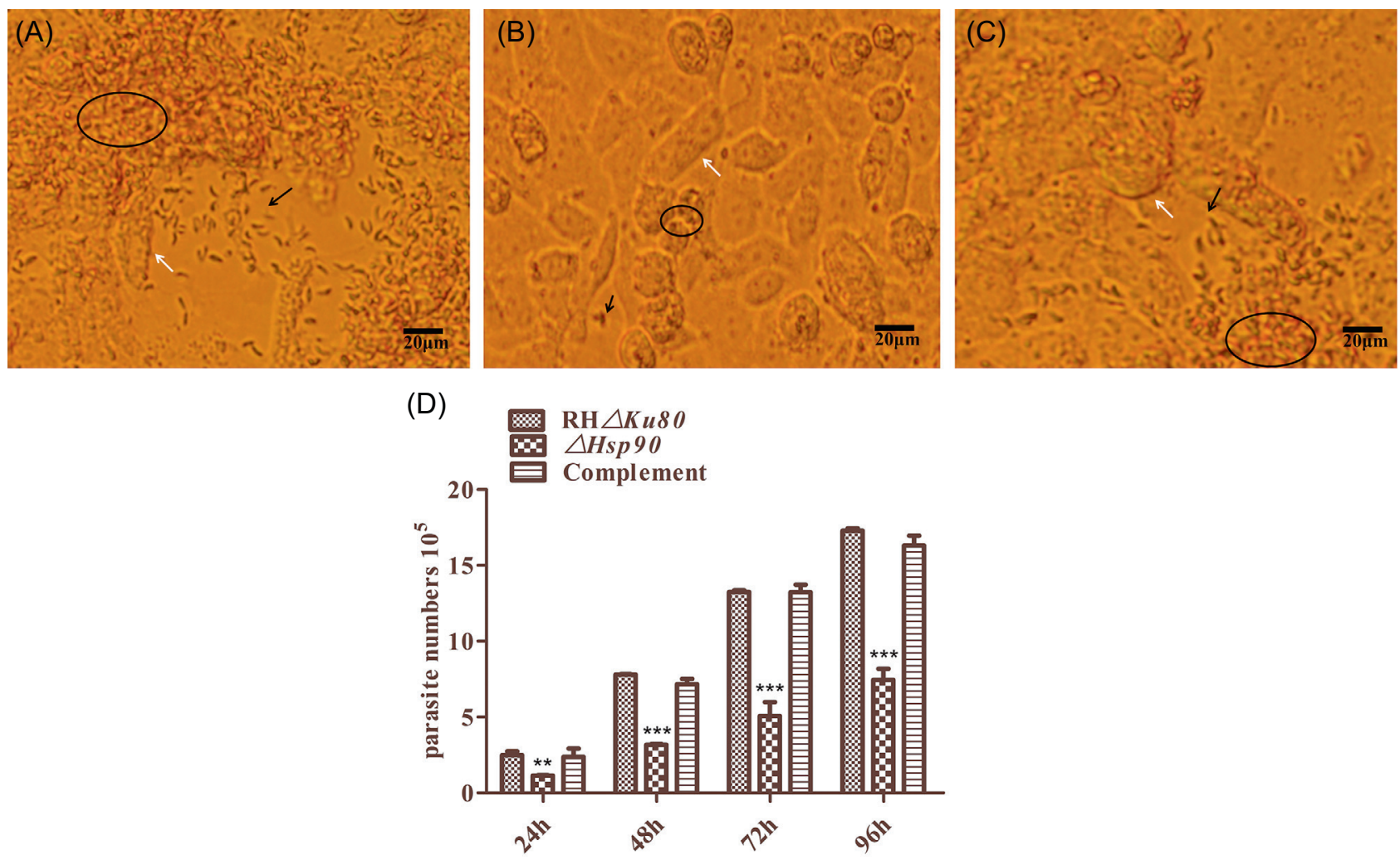

Figure 6. Growth of Hsp90 knockout parasite ( $4 H s p 90)$ in vitro. The parasites were cultured in African green monkey kidney (Vero) cells, $10^{5}$ T. gondii were added to the 6 -well plates, and the infection ratio was $1: 1$. Observation of RH $\Delta k u 80$ (A), $\Delta H s p 90$ (B), and complemented (C) parasites by inverted microscope. The plaque produced by $\triangle P K A R$ strains (Fig. $6 \mathrm{~B}$ ) was significantly smaller than that of RH $\triangle k u 80$ and complemented parasites (Figs. 6A, 6C), scale bar $=20 \mu \mathrm{m}$. T. gondii tachyzoites and Vero cells were indicated by arrows, $T$. gondii (black arrow), Vero cells (white arrow). (D) The parasites were collected at the same time, and genomic DNA was extracted by TIANGEN kit. T. gondii DNA was detected by SYBR-green real-time PCR using B1 primer pairs, the standard curve was obtained by the known concentration of the RH $\Delta k u 80$ parasites with the primers (B1), and the parasite number was calculated by interpolation from this standard curve. $* * p<0.01, * * * p<0.001$.

which was consistent with other research [9]. Specifically, the parasite load in the liver of mice infected with $\mathrm{RH} \Delta k u 80$ parental cells was $1809 / \mathrm{mg}$, whereas that of $\Delta H s p 90$ knockout mice was 91.2 , which was a $19.8 \times$ reduction $(p<0.001)$. The average parasite load in the brain was reduced by 3.2 -fold $(p<0.05)$. Similarly, the parasite loads in the spleen and lungs were also significantly lower in mice receiving knockout parasites than parental cells $(p<0.01)$ (Fig. 8). In contrast, the mice having received the complemented parasites had similar parasite loads to the animals that were infected with parental cells in all four kinds of tissues, i.e. the liver, brain, spleen, and lungs (Fig. 8). Taken together, the results of in vivo infection in a mouse model clearly showed that deletion of the HSP90 gene attenuated T. gondii greatly, as demonstrated by a much longer survival period and a high magnitude reduction in parasite load.

\section{Discussion}

$H s p 90$ proteins of apicomplexan protozoa play a pivotal role in the parasite's biology and virulence. Plasmodium Hsp90 had been shown to regulate parasite growth in human

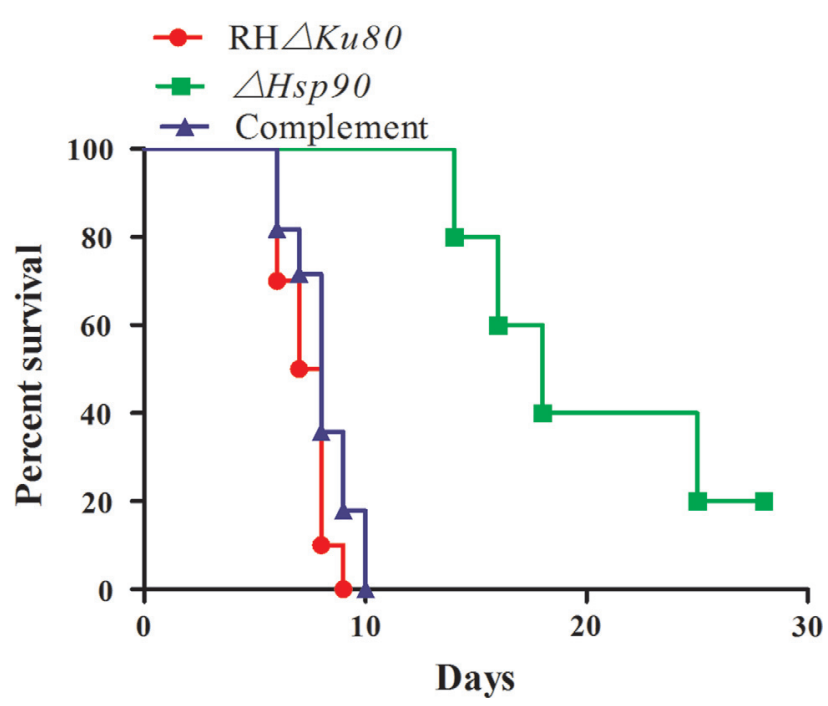

Figure 7. Virulence of $H s p 90$ knockouts in BALB/c mice Purified tachyzoites from different strains were intraperitoneally injected ( $10^{3}$ parasites/mice) into $6-8$ weeks of female BALB/c mice, 10 mice/group; the survival rate was monitored. The experiment was performed three times. 


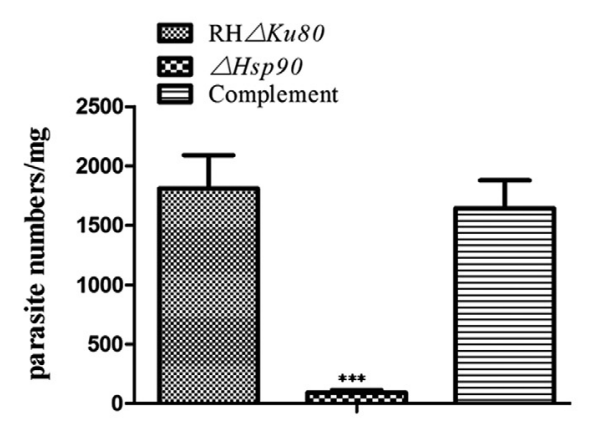

Liver

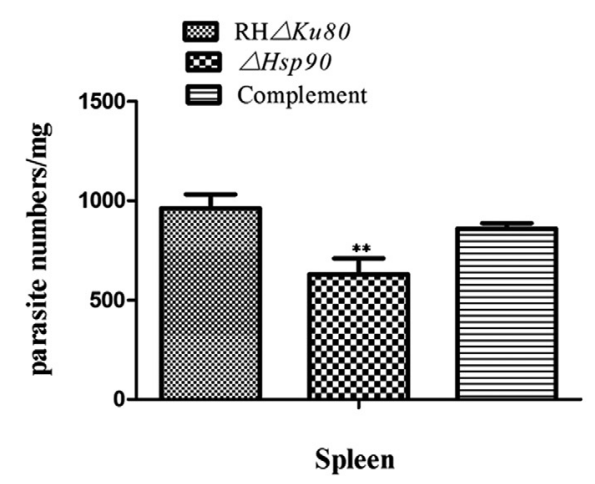

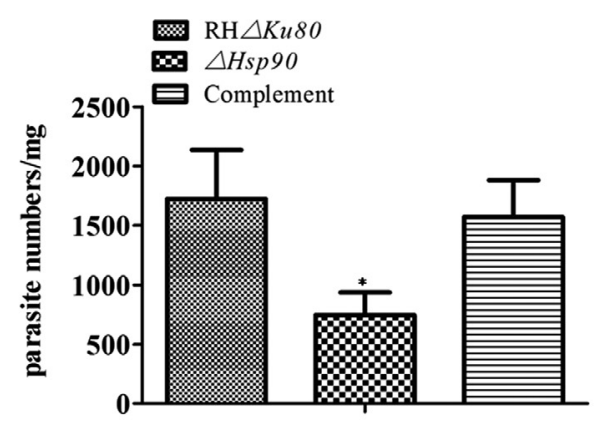

Brain

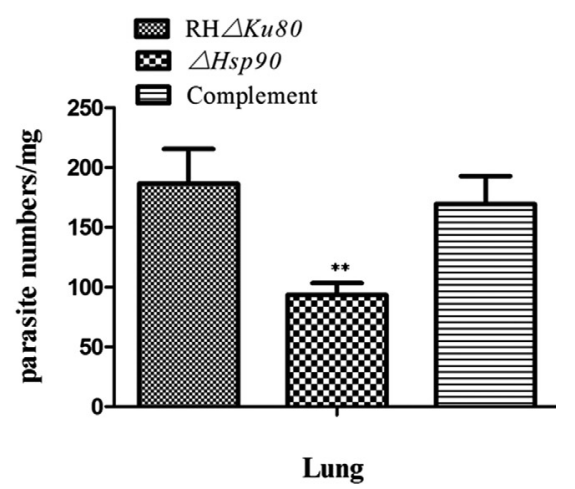

Figure 8. Analysis of parasite burden in BALB/c mice. The parasite burden was also detected in infected mice when the symptoms of T. gondii infection appeared. Parasite burden was detected in liver, brain, spleen, and lung tissues. The data were means $\pm S D$ for three experiments. $* p<0.05, * * p<0.01, * * * p<0.001$.

erythrocytes [52]. Geldanamycin (GA) competitively binds to the N-terminal ATP-binding pocket of Hsp90, leading to the inhibition of its chaperone cycle and reducing the capacity of E. tenella to invade and grow in host cells [38]. Exposure of Babesia gibsoni to heat shock at $43{ }^{\circ} \mathrm{C}$ induced a dramatic increase in its Hsp90 transcripts [53]. Hsp90 is important in other protozoa as well. In Leishmania donovani, Hsp90 is involved in the maintenance of motility and proliferation, and serves as part of the signal transduction pathways that regulates stage-specific gene expression [20, 21, 36]. Interestingly, in closely related Trypanosoma cruzi, Hsp90 was related to response to stress but not stage differentiation [18].

Although the T. gondii Hsp90 gene has been described $[2,13]$, there is very limited information available regarding its biological function. In the current studies, we demonstrated that $T$. gondii Hsp90 plays an important role in bradyzoite differentiation, host-cell invasion, and growth and virulence.

T. gondii interconverts between rapidly dividing tachyzoites and latent encysted bradyzoites. The encysted bradyzoites are critical for the maintenance of $T$. gondii in nature, especially for transmitting the parasite among various intermediate hosts. The interconversion process is accompanied by morphological changes and metabolic adaptations [11, 37]. Toursel and colleagues confirmed that a gene encoding Hsp60 of T. gondii was selectively expressed during the intracellular development of the parasite [49]. The regulatory regions of the $T$. gondii Hsp70 gene were analyzed by a $\beta$-galactosidase expression vector transfected into the $\mathrm{RH}$ strain. At $\mathrm{pH} 8.1$ ( $\mathrm{pH}$ stress), which leads to bradyzoite development, the expression level of Hsp70 was increased [32]. However, the factors and mechanisms governing the interconversion are poorly understood at the molecular level. Studies investigating the switch between the tachyzoite and bradyzoite have confirmed that the SAG1-related sequence (SRS) [27], zinc finger protein (ZFP1) [51], cyclic AMP-dependent protein kinase subunit 3 (PKAc3) [48], mitogen-activated protein kinase 1 (MAPK1) [7], and autophagy-related proteins (Atg) [29] are all involved in bradyzoite differentiation. In the present study, the transcriptional level of the $H s p 90$ gene was determined when T. gondii cells were under stress at $41{ }^{\circ} \mathrm{C}$ or $\mathrm{pH} 8.1$. It was found that $H s p 90$ transcripts increased under these stressful conditions, which was similar to the findings of research carried out by Echeverria et al. [13]. The results indicated that developmental differentiation of $T$. gondii may be governed by both exogenous stress factors and complex endogenous cellular environments.

Recent studies have indicated that Hsp90 is crucial for the survival and growth of parasites. Cellular components involved in these processes include up-regulation in transporters, cysteine proteases, cytoskeletal proteins, and components of the proteasome, just to name a few $[10,47]$. In the Leishmania donovani parasite, the co-chaperone of Hsp90 called P23 acts against various stresses, especially the inhibitory effect of GA, and protects the cells from the harmful effect of the Hsp90 inhibitor, and as a result, the cells grow normally even under such conditions [22]. Other studies of Leishmania donovani have indicated that the proliferation, viability, and infectivity of the kinetoplastid parasite were affected by Hsp90-Sti1 
interaction. Stil is a binding motif of Hsp90. The interaction compound is involved in stage-specific phosphorylation, signal transduction cascades, and the chaperone phosphorylation protein modifications related to parasite viability [21, 36]. In the present study, we demonstrated that deletion of Hsp90 in $T$. gondii made the parasites grow slower in Vero cells than the wild-type parasite $\mathrm{RH} \Delta k u 80$, which indicated that Hsp90 participated in cellular growth, and this process may be accomplished by its interaction with co-chaperones.

In a number of organisms, calcineurin, a calcium-activated protein phosphatase, is important for regulating responses to stresses [47]. It has been shown in murine models that calcineurin is an important virulence factor [4]. Interestingly, S. cerevisiae Hsp90 interacts with calcineurin and Hsp90 increases calcineurin activity in a dose-dependent manner, which may affect the growth and survival of cells [24]. In the current study, we showed that mice receiving intraperitoneal injections of $\Delta H s p 90$ lived much longer than the mice inoculated with either RH $\Delta k u 80$ or complemental parasites. Our data further demonstrated lower parasite loads in tissues of the liver, lungs, spleen, and brain in mice given HSP90 knockout parasites, and that the heaviest parasite loads were found in the liver and brain, which was consistent with a previous report [9].

Previous studies have shown that the heat shock response can cause the dissociation of the IKB kinase (IKK) complex which is the major activator of the NF- $\kappa B$ complex, then inhibits the activation of the NF-kB signaling pathway [45], and inhibits innate immunity or cellular responses in order to help cells to survive tissue injury [40]. Consequently, the function of $T g \mathrm{HSP} 90$ in the growth of $T$. gondii may involve multiple cellular signaling pathways. Future studies of TgHSP90 will be needed to clarify the interaction between HSP90 and the signaling molecules. The T. gondii HSPs are divided into six major families that are grouped according to their molecular weight: small heat shock proteins, Hsp40, Hsp60, Hsp70, Hsp90, and Hsp100. They have significant homologies, suggesting that other heat shock proteins may play an important role in the survival of the Hsp90 knockout parasites, but the specific mechanisms were unknown, and this needs further study.

In conclusion, the present study constructed Toxoplasma gondii Hsp90 (TgHsp90) knockout and complementation strains. Our results show that $\operatorname{TgHsp} 90$ contributes to bradyzoite development, invasion, and replication of $T$. gondii in the host cell, and has an important effect on parasite virulence in vivo. These data will provide a useful basis for further study of the gene function of $T g \mathrm{Hsp} 90$ and for screening drug targets of $T$. gondii.

\section{Conflict of interest}

The authors have no conflict of interest.

Acknowledgements. This work was supported by grants from the Science and Technology Department of Zhejiang (No. 2012C12009-2) and the National Natural Science Foundation of China (No. 31672543).

\section{References}

1. Anderson I, Low JS, Weston S, Weinberger M, Zhyvoloup A, Labokha AA, Corazza G, Kitson RA, Moody CJ, Marcello A, Fassati A. 2014. Heat shock protein 90 controls HIV-1 reactivation. Proceedings of the National Academy of Sciences of the United States of America, 111(15), E1528-E1537.

2. Angel SO, Figueras MJ, Alomar ML, Echeverria PC, Deng B. 2014. Toxoplasma gondii Hsp90: potential roles in essential cellular processes of the parasite. Parasitology, 141(9), 1138-1147.

3. Banumathy G, Singh V, Pavithra SR, Tatu U. 2003. Heat shock protein 90 function is essential for Plasmodium falciparum growth in human erythrocytes. Journal of Biological Chemistry, 278(20), 18336-18345.

4. Blankenship JR, Heitman J. 2005. Calcineurin is required for Candida albicans to survive calcium stress in serum. Infection and Immunity, 73(9), 5767-5774.

5. Buguliskis JS, Brossier F, Shuman J, Sibley LD. 2010. Rhomboid 4 (ROM4) affects the processing of surface adhesins and facilitates host cell invasion by Toxoplasma gondii. PLoS Pathogens, 6(4), e1000858.

6. Calderwood SK, Mambula SS, Gray PJ Jr., Theriault JR. 2007. Extracellular heat shock proteins in cell signaling. FEBS Letters, 581(19), 3689-3694.

7. Cao L, Wang Z, Wang S, Li J, Wang X, Wei F, Liu Q. 2016. Deletion of mitogen-activated protein kinase 1 inhibits development and growth of Toxoplasma gondii. Parasitology Research, 115(2), 797-805.

8. Contini C, Seraceni S, Cultrera R, Incorvaia C, Sebastiani A, Picot S. 2005. Evaluation of a real-time PCR-based assay using the lightcycler system for detection of Toxoplasma gondii bradyzoite genes in blood specimens from patients with toxoplasmic retinochoroiditis. International Journal for Parasitology, 35(3), 275-283.

9. Dadimoghaddam Y, Daryani A, Sharif M, Ahmadpour E, Hossienikhah Z. 2014. Tissue tropism and parasite burden of Toxoplasma gondii RH strain in experimentally infected mice. Asian Pacific Journal of Tropical Medicine, 7(7), 521-524.

10. Debnath A, Shahinas D, Bryant C, Hirata K, Miyamoto Y, Hwang G, Gut J, Renslo AR, Pillai DR, Eckmann L, Reed SL, McKerrow JH. 2014. Hsp90 inhibitors as new leads to target parasitic diarrheal diseases. Antimicrobical Agents and Chemotherapy, 58(7), 4138-4144.

11. Dzierszinski F, Nishi M, Ouko L, Roos DS. 2004. Dynamics of Toxoplasma gondii differentiation. Eukaryotic Cell, 3(4), 992-1003.

12. Eaton MS, Weiss LM, Kim K. 2006. Cyclic nucleotide kinases and tachyzoite-bradyzoite transition in Toxoplasma gondii. International Journal for Parasitology, 36(1), 107-114.

13. Echeverria PC, Matrajt M, Harb OS, Zappia MP, Costas MA, Roos DS, Dubremetz JF, Angel SO. 2005. Toxoplasma gondii Hsp90 is a potential drug target whose expression and subcellular localization are developmentally regulated. Journal of Molecular Biology, 350(4), 723-734.

14. Fallahi S, Seyyed Tabaei SJ, Pournia Y, Zebardast N, Kazemi B. 2014. Comparison of loop-mediated isothermal amplification (LAMP) and nested-PCR assay targeting the RE and B1 gene for detection of Toxoplasma gondii in blood samples of children with leukaemia. Diagnostic Microbiology and Infectious Disease, 79(3), 347-354. 
15. Faya N, Penkler DL, Tastan Bishop O. 2015. Human, vector and parasite Hsp90 proteins: a comparative bioinformatics analysis. FEBS Open Bio, 14(5), 916-927.

16. Ferreira da Silva MDF, Barbosa HS, Gross U, Luder CG. 2008. Stress-related and spontaneous stage differentiation of Toxoplasma gondii. Molecular Biosystems, 4(8), 824-834.

17. Galvan-Ramirez Mde L, Troyo-Sanroman R, Roman S, BernalRedondo R, Vazquez Castellanos JL. 2012. Prevalence of Toxoplasma infection in Mexican newborns and children: a systematic review from 1954 to 2009. ISRN Pediatrocs, 2012, 501216.

18. Graefe SEB, Wiesgigl M, Gaworski I, Macdonald A, Clos J. 2002. Inhibition of HSP90 in Trypanosoma cruzi induces a stress response but no stage differentiation. Eukaryotic Cell, 1(6), 936-943.

19. Hartl FU, Hayer-Hartl M. 2002. Molecular chaperones in the cytosol: from nascent chain to folded protein. Science, 295(5561), 1852-1858.

20. Hombach A, Clos J. 2014. No stress-Hsp90 and signal transduction in Leishmania. Parasitology, 141(9), 1156-1166.

21. Hombach A, Ommen G, Chrobak M, Clos J. 2013. The Hsp90Sti1 interaction is critical for Leishmania donovani proliferation in both life cycle stages. Cellular Microbiology, 15(4), 585-600.

22. Hombach A, Ommen G, Sattler V, Clos J. 2015. Leishmania donovani P23 protects parasites against HSP90 inhibitormediated growth arrest. Cell Stress Chaperones, 20(4), 673-685.

23. Hunter CA, Sibley LD. 2012. Modulation of innate immunity by Toxoplasma gondii virulence effectors. Nature Reviews Microbiology, 10(11), 766-778.

24. Imai J, Yahara I. 2000. Role of HSP90 in salt stress tolerance via stabilization and regulation of calcineurin. Molecular Biology of the Cell, 20(24), 9262-9270.

25. Joly AL, Wettstein G, Mignot G, Ghiringhelli F, Garrido C. 2010. Dual role of heat shock proteins as regulators of apoptosis and innate immunity. Journal of Innate Immunity, 2(3), 238-247.

26. Jones JL, Dubey JP. 2012. Foodborne toxoplasmosis. Clinical Infectious Diseases, 55(6), 845-851.

27. Kim SK, Karasov A, Boothroyd JC. 2007. Bradyzoite-specific surface antigen SRS9 plays a role in maintaining Toxoplasma gondii persistence in the brain and in host control of parasite replication in the intestine. Infection and immunity, 75(4), 1626-1634.

28. Lanneau D, de Thonel A, Maurel S, Didelot C, Garrido C. 2007. Apoptosis versus cell differentiation: role of heat shock proteins HSP90, HSP70 and HSP27. Prion, 1(1), 53-60.

29. Li X, Chen DI, Hua Q, Wan Y, Zheng L, Liu Y, Lin J, Pan C, $\mathrm{Hu} \mathrm{X}$, Tan F. 2016. Induction of autophagy interferes the tachyzoite to bradyzoite transformation of Toxoplasma gondii. Parasitology, 143(5), 639-645.

30. Lianos GD, Alexiou GA, Mangano A, Mangano A, Rausei S, Boni L, Dionigi G, Roukos DH. 2015. The role of heat shock proteins in cancer. Cancer Letters, 360(2), 114-118.

31. Liu Q, Wang ZD, Huang SY, Zhu XQ. 2015. Diagnosis of toxoplasmosis and typing of Toxoplasma gondii. Parasite Vector, 28(8), 292.

32. Ma YF, Zhang Y, Kim K, Weiss LM. 2004. Identification and characterisation of a regulatory region in the Toxoplasma gondii hsp70 genomic locus. International Journal for Parasitology, 34(3), 333-346.
33. Messina M, Niesman I, Mercier C, Sibley LD. 1995. Stable DNA transformation of Toxoplasma gondii using phleomycin selection. Gene, 165(2), 213-217.

34. Mohammed SB, Bakheit MA, Ernst M, Ahmed JS, Seitzer U. 2013. Identification and characterization of Theileria annulata heat-shock protein 90 (HSP90) isoforms. Transboundary Emerging Diseases, 60(2), 137-149.

35. Monzote L, Siddiq A. 2011. Drug development to protozoan diseases. Open Medicinal Chemistry Journal, 5, 1-3.

36. Morales MA, Watanabe R, Dacher M, Chafey P, Osorio y Fortea J, Scott DA, Beverley SM, Ommen G, Clos J, Hem S, Lenormand P, Rousselle JC, Namane A, Spath GF. 2010. Phosphoproteome dynamics reveal heat-shock protein complexes specific to the Leishmania donovani infectious stage. Proceedings of the National Academy of Sciences of the United States of America, 107(18), 8381-8386.

37. Paredes-Santos TC, Tomita T, Yan Fen M, de Souza W, Attias M, Vommaro RC, Weiss LM. 2016. Development of dual fluorescent stage specific reporter strain of Toxoplasma gondii to follow tachyzoite and bradyzoite development in vitro and in vivo. Microbes and Infection, 18(1), 39-47.

38. Peroval M, Pery P, Labbe M. 2006. The heat shock protein 90 of Eimeria tenella is essential for invasion of host cell and schizont growth. International Journal for Parasitology, 36(10-11), 1205-1215.

39. Radke JR, Guerini MN, Jerome M, White MW. 2003. A change in the premitotic period of the cell cycle is associated with bradyzoite differentiation in Toxoplasma gondii. Molecular and Biochemical Parasitology, 131(2), 119-127.

40. Richa A, Moushami M, Subhash CL. 2007. Heat shock genes integrating cell survival and death. Journal of Biosciences, 32(3), 595-610.

41. Robert-Gangneux F. 2014. It is not only the cat that did it: how to prevent and treat congenital toxoplasmosis. Journal of Infection, 68(1), S125-S133.

42. Roesch F, Meziane O, Kula A, Nisole SB, Fo Porrot, Anderson I, Mammano F, Fassati A, Marcello A, Benkirane M, Schwartz O. 2012. Hyperthermia stimulates HIV-1 replication. PloS Pathogens, 8(7), e1002792.

43. Rommereim LM, Hortua Triana MA, Falla A, Sanders KL, Guevara RB, Bzik DJ, Fox BA. 2013. Genetic manipulation in $\triangle k u 80$ strains for functional genomic analysis of Toxoplasma gondii. Journal of Visualized Experiments, 77, 50598.

44. Roy N, Nageshan RK, Ranade S, Tatu U. 2012. Heat shock protein 90 from neglected protozoan parasites. Biochimica Biophysica Acta-biomembranes, 1823(3), 707-711.

45. Salminen A, Paimela T, Suuronen T, Kaarniranta K. 2008. Innate immunity meets with cellular stress at the IKK complex: regulation of the IKK complex by HSP70 and HSP90. Immunology Letters, 117(1), 9-15.

46. Silva NM, Tafuri WL, Alvarez-Leite JI, Mineo JR, Gazzinelli RT. 2002. Toxoplasma gondii in vivo expression of BAG-5 and cyst formation is independent of TNF p55 receptor and inducible nitric oxide synthase functions. Microbes and Infection, 4(3), 261-270.

47. Singh M, Sharma S, Bhattacharya A, Tatu U. 2015. Heat Shock Protein 90 regulates encystation in Entamoeba. Frontiers in Microbiology, 6, 1125.

48. Sugi T, Ma YF, Tomita T, Murakoshi F, Eaton MS, Yakubu R, Han B, Tu V, Kato K, Kawazu S, Gupta N, Suvorova ES, White MW, Kim K, Weiss LM. 2016. Toxoplasma gondii cyclic AMP-dependent protein kinase subunit 3 is involved in the 
switch from tachyzoite to bradyzoite development. MBio, 7(3), e00755-16.

49. Toursel C, Dzierszinski F, Bernigaud A, Mortuaire M, Tomavo S. 2000. Molecular cloning, organellar targeting and developmental expression of mitochondrial chaperone HSP60 in Toxoplasma gondii. Molecular and Biochemical Parasitology, 111(2), 319-332.

50. Tsan MF, Gao B. 2009. Heat shock proteins and immune system. Journal of Leukocyte Biology, 85(6), 905-910.

51. Vanchinathan P, Brewer JL, Harb OS, Boothroyd JC, Singh U. 2005. Disruption of a locus encoding a nucleolar zinc finger protein decreases tachyzoite-to-bradyzoite differentiation in Toxoplasma gondii. Infection immunology, 73(10), 6680-6688.

52. Wang T, Maser P, Picard D. 2016. Inhibition of Plasmodium falciparum Hsp90 contributes to the antimalarial activities of aminoalcohol-carbazoles. Journal of Medicinal Chemistry, 59(13), 6344-6352.

53. Yamasaki M, Tsuboi $Y$, Taniyama $Y$, Uchida N, Sato R, Nakamura K, Ohta H, Takiguchi M. 2016. Molecular cloning, phylogenetic analysis and heat shock response of Babesia. Journal of Veterinary Medical Science, 78(8), 1355-1360.

54. Zhang H, Zhang Y, Cao J, Zhou Y, Wang N, Zhou J. 2013. Determination of stage interconversion in vitro and in vivo by construction of transgenic Toxoplasma gondii that stably express stage-specific fluorescent proteins. Experimental Parasitology, 134(3), 275-280.

55. Zheng J, Jia H, Zheng Y. 2015. Knockout of leucine aminopeptidase in Toxoplasma gondii using CRISPR/Cas9. International Journal for Parasitology, 45(2-3), 141-148.

Cite this article as: Sun H, Zhuo X, Zhao X, Yang Y, Chen X, Yao C \& Du A: The heat shock protein 90 of Toxoplasma gondii is essential for invasion of host cells and tachyzoite growth. Parasite, 2017, 24, 22.

\section{-0 PARASTE}

An international open-access, peer-reviewed, online journal publishing high quality papers on all aspects of human and animal parasitology

Reviews, articles and short notes may be submitted. Fields include, but are not limited to: general, medical and veterinary parasitology; morphology, including ultrastructure; parasite systematics, including entomology, acarology, helminthology and protistology, and molecular analyses; molecular biology and biochemistry; immunology of parasitic diseases; host-parasite relationships; ecology and life history of parasites; epidemiology; therapeutics; new diagnostic tools.

All papers in Parasite are published in English. Manuscripts should have a broad interest and must not have been published or submitted elsewhere. No limit is imposed on the length of manuscripts.

Parasite (open-access) continues Parasite (print and online editions, 1994-2012) and Annales de Parasitologie Humaine et Comparée (1923-1993) and is the official journal of the Société Française de Parasitologie. 